\title{
Trabajos de investigación
}




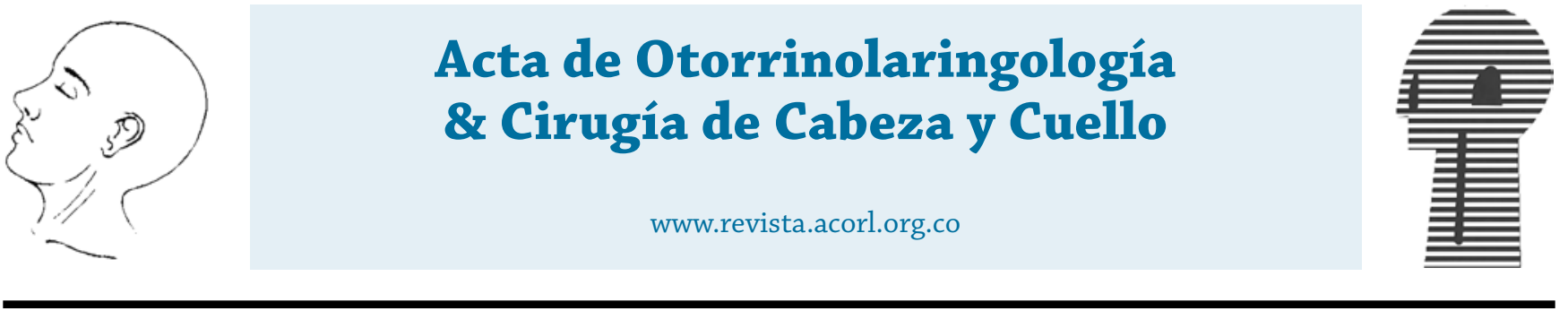

Trabajos de investigación

\title{
Tratamiento quirúrgico del síndrome de apnea hipopnea obstructiva del sueño en la Clínica Rivas, Bogotá - Colombia Surgical treatment of obstructive sleep apnea hypopnea syndrome in the Rivas Clinic, Bogota - Colombia
}

\author{
Steve Amado Galeano MD*, Juan Fernando Gómez Lopera MD**, Diego Andrés Corredor Zuluaga***, \\ Adriana Carolina Navarro Navarro**** \\ Clínica de Apnea y Medicina del Sueño. Clínica Rivas, universidad del Rosario. Bogotá D.C. \\ * Otorrinolaringólogo Universidad del Rosario, Hospital Universitario de la Samaritana. Clínica de Apnea y Medicina del Sueño \\ Centro de Diagnóstico de Sueño, Bogotá. Clínica de Apnea y Medicina del Sueño. Clínica Rivas, Bogotá. \\ ** Residente Otorrinolaringología Universidad del Rosario - Hospital Universitario de la Samaritana. Bogotá D.C. \\ *** Residente Otorrinolaringología Universidad del Rosario - Hospital Universitario de la Samaritana. Bogotá D.C. \\ **** Médica Otorrinolaringóloga - Epidemióloga Universidad Nacional de Colombia - Universidad del Bosque, Clínica Rivas.
}

Forma de Citar: Amado YS, Gómez JF, Corredor DA, Navarro AC. Tratamiento quirúrgico del síndrome de apnea hipopnea obstructiva del sueño en la clinica rivas, Bogotá - Colombia. Acta otorrinolaringol. cir. cabeza cuello; 2015;43(2):93-100.

INFORMACIÓN DEL ARTÍCULO

Historia del artículo:

Recibido: 31 de marzo de 2014

Revisado: 04 de junio de 2014

Aceptado: 12 de agosto de 2014

Palabras clave:

Apna del Sueño Obstructiva, Polisomnografía, Cirugía, Calidad de Vida.

\section{RESUMEN}

Introducción: El Síndrome de Apnea Hipopnea Obstructiva del Sueño es un trastorno respiratorio del sueño mayor ampliamente conocido, con importantes implicaciones para los pacientes y cuya incidencia ha venido en aumento durante los últimos años; comprende diversas manifestaciones clínicas que varían desde el ronquido hasta consecuencias cardiovasculares importantes. Objetivo: Describir la experiencia de los procedimientos quirúrgicos más utilizados para el tratamiento de pacientes con Trastornos Respiratorios del Sueño en la Clínica Rivas. Diseño: Estudio observacional descriptivo. Métodos: Revisión de 366 historias clínicas de pacientes con diagnóstico clínico y Polisomnográfico de SAHOS intervenidos quirúrgicamente debido al Trastorno Respiratorio del Sueño por rechazo de terapia de presión positiva en 3 años de observación. Resultados: Se evaluaron diferencias en medianas de los cambios del IAH, índice de Saturación de oxigeno basal y mínima, y el índice de

Correspondencia:

Steve Amado Galeano,

steveamadog@gmil.com

Clínica Rivas, Avenida K 19 No. 100 - 88, Bogotá, Colombia

Tel. (57-1) 57 (1) 6163077 
microdespertares nocturnos tanto prequirúrgica como postquirúrgicamente. Como medida de evaluación secundaria se evaluaron las complicaciones quirúrgicas. Conclusión: En nuestra institución, como centro de referencia en apnea del sueño, la cirugía ha demostrado que disminuye de forma significativa gravedad del SAHOS y disminuye el riesgo de los pacientes con trastornos respiratorios del sueño que han rechazado el dispositivo de presión positiva.

\section{ABSTRACT}

Key words:

Sleep Apnea, Obstructive; Polysomnography; Surgery; Quality of life.
Introduction: Obstructive Sleep Apnea Hypopnea Syndrome is a major sleep breathing disorder widely known, with important implications for patients whose incidence has been increasing in recent years; comprises various clinical manifestations ranging from snoring to major cardiovascular consequences. Objective: To describe the experience of surgical procedures commonly used for the treatment of patients with respiratory sleep disorders in Rivas Clinic. Design: Descriptive study. Methods: A review of medical records of 366 patients with clinical and polysomnographic diagnosis of OSAHS and underwent surgically due to Sleep Respiratory Disorder for rejection of positive pressure therapy in 3 years of observation was performed. Results: Differences in median changes in Apnea Hipopnea Index, baseline saturation index and minimum oxygen, and index of nocturnal arousals both preoperative and postoperatively were evaluated. As a secondary assessment of surgical complications were evaluated. Conclusion: At our institution, as a reference center on sleep apnea, surgery has been shown to decrease significantly OSAHS severity and decreases the risk of patients with respiratory sleep disorders that have rejected positive pressure device.

\section{Introducción}

El Síndrome de Apnea Hipopnea Obstructiva del Sueño (SAHOS) es un trastorno respiratorio del sueño (TRS) mayor ampliamente conocido, con importantes implicaciones para los pacientes y cuya incidencia ha venido en aumento durante los últimos años. Su prevalencia en personas de mediana edad es del $4 \%$ en hombres y del $2 \%$ en mujeres. Sin embargo, las cifras sugieren que hasta el $26 \%$ de los adultos están en alto riesgo de sufrir SAHOS $(1,2)$. A pesar de ello, los métodos diagnósticos estructurales disponibles todavía dejan un vacío dentro de la valoración integral de la vía aérea superior del paciente con SAHOS y más aún a la hora de definir su manejo quirúrgico $(1,2,3)$.

El SAHOS, comprende diversas manifestaciones clínicas que varían desde el ronquido hasta consecuencias cardiovasculares importantes, secundarias a episodios repetitivos de obstrucción de la vía aérea superior, que provoca constantes desaturaciones y microdespertares transitorios, que dan lugar a un sueño fragmentado y poco reparador $(3,4)$.

El primer tratamiento eficaz que se describió en los adultos con SAHOS fue la traqueostomía, la cual ofrece un porcentaje de curación del $100 \%$. Sin embargo, debido a la morbilidad significativa asociada con este procedimiento quirúrgico se adoptaron varios tratamientos alternativos $(4,5,6)$.

Sullivan \& colaboradores en 1981, describieron el Dispositivo de Presión Positiva Continua sobre la vía aérea
(CPAP) como un tratamiento altamente eficaz para SAHOS. Desde entonces, el tratamiento médico con CPAP se considera como la terapia de primera línea y piedra angular del tratamiento, por lo tanto, a cada paciente siempre se le debe ofrecer el tratamiento con CPAP antes de la cirugía. El tratamiento quirúrgico multinivel para SAHOS es generalmente reservado para aquellos pacientes que han fracasado con intervenciones médicas $(4,7)$.

Para llegar a una adecuada predicción del resultado quirúrgico de los pacientes con SAHOS, es necesario realizar una minuciosa evaluación de la vía aérea superior de estos pacientes y descartar todas las etiologías posibles en un mismo paciente.

La cirugía puede ser utilizada como una opción de tratamiento primario en pacientes seleccionados que tienen problemas anatómicos identificables o se puede utilizar como una opción de tratamiento de "rescate" para los pacientes que no mejoran con la terapia de elección con CPAP o más importante aún en pacientes que rechazan de forma enfática la terapia de presión positiva $(5,8)$.

A pesar de una tasa de curación variables, la cirugía ha demostrado que disminuye de forma rutinaria gravedad del SAHOS y aumenta la calidad de vida del paciente.

El tratamiento quirúrgico para el SAHOS tiene como objetivo mejorar la permeabilidad de la vía aérea superior, abordando el sitio de obstrucción seleccionado e identificado previamente. Usualmente son más de una las áreas que pue- 
den ser responsables de la reducción del calibre de la vía aérea implicadas tanto en zona de colapso como en zona de vibración y producción de sonido, también se han desarrollado diferentes técnicas quirúrgicas (6,9-11). Como consecuencia de la etiología multifactorial y heterogénea del SAHOS, las técnicas quirúrgicas deben ser seleccionadas y realizadas específicamente para cada paciente, de esta forma se logra individualizar cada caso por tipo de apnea y tipo de obstrucción.

$\mathrm{Al}$ igual que con cualquier otro tipo de tratamiento, para el SAHOS, los tratamientos quirúrgicos tienen una eficacia variable, pero son una herramienta muy importante para esta patología en pacientes seleccionados y ha demostrado tener eficacia en la disminución de la morbilidad y mortalidad asociadas a la enfermedad $(1,12)$.

La eficacia de procedimientos aislados para SAHOS tienen porcentajes de éxito variables del 40 al 85\%, siendo más efectivas las técnicas multinivel y con individualización de pacientes $(7,13-15)$.

El objetivo principal del tratamiento quirúrgico es reducir o eliminar el colapso de las vías respiratorias que se produce durante el sueño, mientras se preservan las funciones normales de la vía aéreo-digestiva superior y de las estructuras relacionadas, tales como el habla y la deglución. En la literatura, el éxito de la cirugía se ha definido tradicionalmente como una reducción del IAH (Índice de apneas-hipopneas) basal en un $50 \%$ y un IAH $<20$ después de la cirugía. Los criterios para una cura de tratamiento se definen como un IAH $<5$ después de la cirugía. Otros objetivos de la cirugía incluyen la normalización de la calidad del sueño, la mejora del IAH y los niveles de saturación de oxígeno $(5,16,17)$. Las quejas del compañero de cama con los ronquidos también deben ser tenidas en cuenta como un resultado final importante.

En nuestro medio, en la consulta de la Clínica de Apnea de la Clínica José A. Rivas, atendemos aproximadamente entre 10 y 25 pacientes diarios con diagnóstico de SAHOS. La prevalencia de la enfermedad en Colombia se encuentra alrededor de $25 \%$ en hombres y $10 \%$ de las mujeres. Estos pacientes requieren en promedio de 63 meses entre consultas y exámenes paraclínicos por año para obtener un diagnóstico adecuado, teniendo cada uno de estos estudios un costo total aproximado de $\$ 800.000$ pesos $(8,18-20)$.

Debido a que el SAHOS es una patología multifactorial, los tratamientos son poco eficaces a causa de la insuficiente evaluación de la vía aérea superior. Para ello es importante realizar una adecuada predicción del resultado quirúrgico realizando una minuciosa evaluación de la vía aérea superior y descartando todas las etiologías posibles, lo que permitirá obtener mejores resultados al optar por un manejo quirúrgico multinivel según sea el caso $(10,11,21-23)$.

Los protocolos de manejo en nuestra institución tienen como mínimo una videofibronasolaringoscopia dinámica con paciente acostado y sentado, maniobras dinámicas de ronquido, polisomnografía basal y con su respectiva titulación de CPAP, estudio hormonal y metabólico, y en pacientes seleccionados estudios de comorbilidad cardiopulmonar.
La finalidad del presente estudio es describir los procedimientos quirúrgicos realizados en nuestra institución seleccionados mediante nuestro protocolo diagnóstico para el tratamiento quirúrgico multinivel en varias etapas del Síndrome de Apnea Hipopnea Obstructiva del Sueño, debido a que hasta la fecha existen muy pocos estudios en nuestro medio que evalúen la efectividad de este tipo de tratamientos en el manejo de la enfermedad y comparen sus resultados con la literatura ya reportada.

\section{Materiales y métodos}

Se realizó un estudio descriptivo retrospectivo. Se revisaron las historias clínicas de los pacientes atendidos en la consulta de la Clínica de Apnea y Medicina del Sueño de la Clínica José A. Rivas entre Enero de 2011 y Enero de 2014, con diagnóstico de Síndrome de Apnea Hipopnea Obstructiva del Sueño que cumplieron con los criterios de inclusión y que fueron sometidos a algún procedimiento quirúrgico por enfermedad asociada causante de obstrucción en cualquier nivel de la vía aérea superior.

Los criterios de inclusión eran: 1) Pacientes con diagnóstico de Síndrome de Apnea Hipopnea Obstructiva del Sueño confirmado por Polisomnografía e historia clínica. 2) sometidos a algún procedimiento quirúrgico por obstrucción en cualquier nivel de la vía aérea superior. 3) a quienes se les realizó un adecuado seguimiento de estudios pre y posoperatorio comparables. 4) Pacientes con rechazo a terapia de presión positiva.

Mientras que los criterios de exclusión fueron: 1) Pacientes en los que no se encontraron en la revisión de la historia clínica la documentación necesaria, y 2) Pacientes con diagnóstico de trastorno respiratorio del sueño que no presentaron datos completos para mediciones pre y postquirúrgicas de IAH, índice de Saturación e índice de microdespertares nocturnos en la historia clínica.

Se evaluaron diferencias en medianas de los cambios del IAH, Saturación de oxigeno e índice de microdespertares nocturnos prequirúrgicos y postquirúrgicos de los pacientes sometidos a procedimientos quirúrgicos. Como medida de evaluación secundaria se evaluaron las complicaciones quirúrgicas.

Se analizó con el programa Stata 11, en total fueron validas 256 muestras, con datos completos de IAH pre y post quirúrgico. Se realizó prueba de distribución normal de Shapiro wilk, obteniendo que las muestras no tienen una distribución normal, por lo que se utilizó prueba de Willcoxon para diferencia de medianas, de las muestras pareadas obteniendo una $\mathrm{p}<0,00001$.

\section{Resultados}

En la institución donde se realizó el estudio, consultaron por primera vez un total de 6542 pacientes a la clínica de medicina del sueño entre los periodos comprendidos por las fechas 01 de Enero de 2010 al 28 de Febrero del 2014. Del 
total de pacientes que consultaron, el 52\% (3401 pacientes) eran mujeres, mientras que el $48 \%$ (3141 pacientes) eran hombres.

De los 6542 pacientes valorados, el $98 \%$ tuvo diagnóstico polisomnográfico de TRS, y el 100\% tuvo previamente una valoración y manejo multidisciplinario por especialidades médicas afines, entre las que se encuentran Otorrinolaringología, Neumología, Cardiología, Cirugía Oral y Maxilofacial, Pediatría y Medicina Interna.

El promedio de edad fue de 38.1 años, con un rango de edad entre los 3 años y los 77 años.

De acuerdo con la Asociación Americana de Pediatría, la población pediátrica se definió como pacientes menores de 15 años de edad.

Aquellos pacientes mayores de los 15 años, fueron definidos, valorados y tratados para efectos prácticos como adultos.

El total de pacientes pediátricos valorados inicialmente por consulta externa fueron 659 , que corresponde al $10.07 \%$ del total de pacientes que consultaron (659/6542). Mientras que el número de adultos corresponde al $89,9 \%$ del total de pacientes (5883/6542).

Con respecto a la población pediátrica, de los 659 pacientes, 119 fueron sometidos a procedimientos quirúrgicos como parte del tratamiento del TRS, que corresponde al $18.05 \%$ de los pacientes pediátricos. En cuanto a su distribución por sexo, $59,6 \%$ fueron niños (71 pacientes) y $40,33 \%$ (48 pacientes) eran niñas.

Del total de población pediátrica (119 pacientes) que fue sometida a algún procedimiento quirúrgico, el $100 \%$ presentaban diagnóstico SAHOS realizado por polisomnografía, de los cuales tomando como criterio el índice de severidad de la Asociación Americana de Medicina del Sueño, se distribuyeron según su severidad de la siguiente manera: 3 (2,52\%) Leve: (IAH 1 - 5/Hora); 39 (32,77\%) Moderado: (IAH 5 - 15/Hora); y 74 (62,18\%) Severo: (IAH >15/Hora) (Gráfico 1).

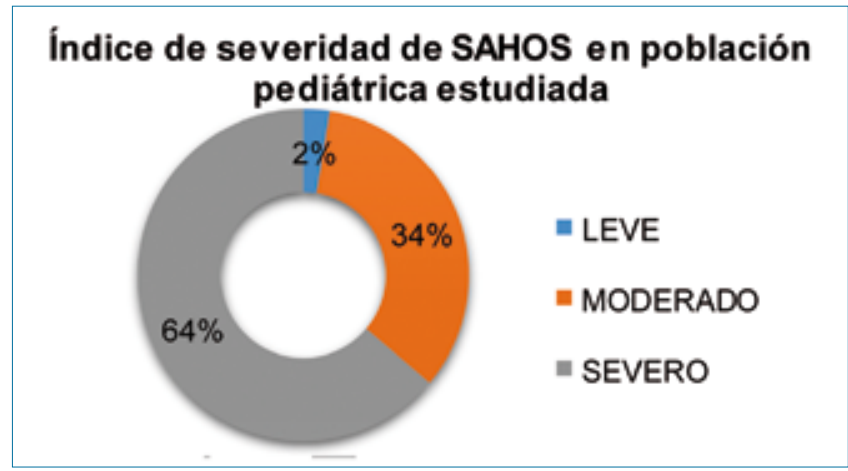

Gráfica 1. Índice de severidad de SAHOS en la población pediátrica estudiada.

El procedimiento quirúrgico que más se realizó en la población pediátrica fue la Adenoamigdalectomía (77 pacientes), que corresponden al $64,70 \%$ de los pacientes pediátricos intervenidos quirúrgicamente, seguido de Amigdalectomía en 8 pacientes $(6,72 \%)$, Radiofrecuencia de base de lengua en $15(12,6 \%)$, Adenoidectomía en $6(5,04 \%)$, Turbinoplastia en 5 (4,20\%), y otros 19,3\%. (Gráfico 2).

De los 6542 pacientes que consultaron al servicio de consulta externa de Medicina del Sueño, 366 pacientes fueron sometidos a procedimientos quirúrgicos, de los cuales 119 fueron pediátricos y los restantes 247 adultos.

En total 247 hombres $(67,49 \%)$ y 119 mujeres $(32,51 \%)$, cumplieron con los criterios de inclusión para el estudio.

El Síndrome de apnea hipopnea obstructiva del sueño (SAHOS), fue el diagnóstico común para los pacientes incluidos en la investigación.

Otros diagnósticos asociados a SAHOS que se identificaron concomitantemente fueron: Síndrome de Down (2 pacientes), Síndrome de Goldenhar (1 paciente), Síndrome de Crouzon (1 paciente), déficit pituitario (1 paciente) y translocación del cromosoma 8 y 12 (1 paciente).

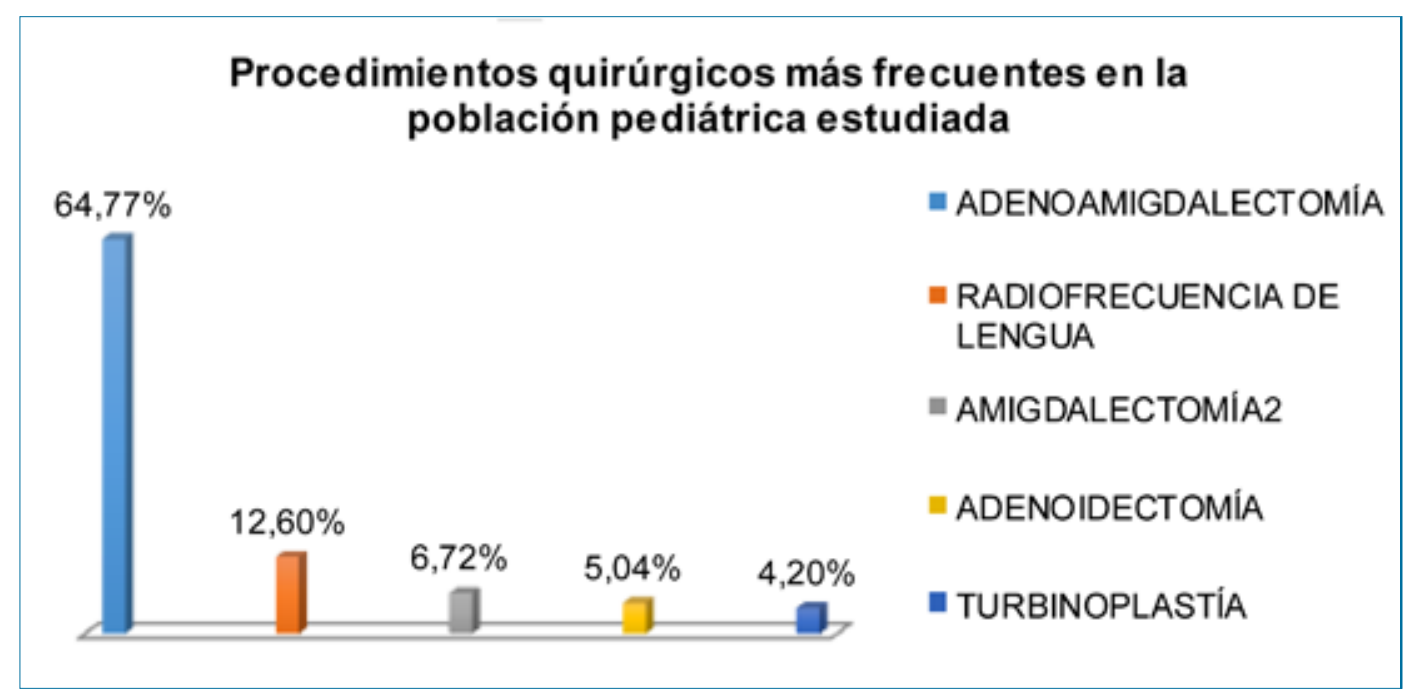

Gráfica 2. Procedimientos quirúrgicos realizados más frecuentemente en la población pediátrica en Clínica de medicina del sueño, Clínica Rivas. 
Se practicó nasofibrolaringoscopia en el $85 \%$ de los pacientes pediátricos y en el $100 \%$ de los pacientes adultos sometidos a procedimientos quirúrgicos como tratamiento para el TRS. Como estudio complementario, se realizó nasofibrolaringoscopia; en los hallazgos de esta, solo 20 pacientes $(0,64 \%)$ presentaban un único sitio anatómico de obstrucción. En términos generales, los sitios de obstrucción son múltiples y mixtos.

Del total de pacientes, 62 requirieron que se les practicará DISE (Endoscopia inducida por medicamentos), con el fin de clarificar o identificar el sitio de obstrucción causante del trastorno respiratorio del sueño.

Con respecto a la población adulta 247 pacientes fueron sometidos a algún procedimiento quirúrgico como manejo del trastorno respiratorio del sueño. Entre los pacientes señalados, la distribución según el índice de severidad estipulado por la Asociación Americana de Medicina del Sueño: 40 pacientes $(16,19 \%)$ presentaron SAHOS leve (IAH 5 - 15/ Hora), 69 pacientes $(27,93 \%)$ presentaron SAHOS Moderado (IAH 15 - 30/ Hora), y 138 pacientes $(55,8 \%)$ presentaron SAHOS Severo (IAH > 30/Hora). (Gráfico 3).

\section{Índice de severidad de SAHOS en población adulta estudiada}

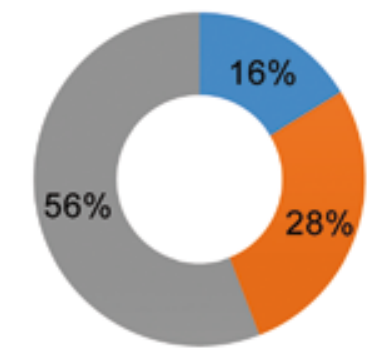

- LEVE

= MODERADO

" SEVERO

Gráfica 3. Índice de severidad de SAHOS en la población adulta estudiada.

Se practicaron más de 22 tipos de procedimientos de forma individual o combinada según las necesidades específicas del caso sujeto, generando procedimientos combinados en 71 diferentes posibilidades de intervención quirúrgica.

La combinación más común fue septoplastia asociado a Turbinoplastia con un total de 101 pacientes correspondiendo a 27.6\%, seguido por Adenoamigdalectomía en 66 pacientes $(18 \%)$. De igual forma, se realizó Amigdalectomía, faringoplastia lateral y radiofrecuencia de lengua de forma simultánea en 16 pacientes (4,4\%); faringoplastia lateral y radiofrecuencia de lengua en 11 pacientes (3\%); y Amigdalectomía en 10 pacientes $(2,7 \%)$.

Se realizó septoturbinoplastia, Amigdalectomía, faringoplastia lateral y radiofrecuencia de lengua en 9 pacientes $(2,45 \%)$. Se practicó Adenoidectomía en el 1,6\% (6 pacientes). La asociación de septoturbinoplastia y cirugía endoscópica fue realizada en un mismo tiempo quirúrgico en 5 pacientes $(1,3 \%)$. De forma pionera en Latinoamérica, como tratamiento para el SAHOS, se realizó implante del nervio hipogloso en 3 pacientes, correspondiendo al 1,21\% de la población sometida a intervención quirúrgica.

Es relevante destacar que de un total de 6542 pacientes, $422(6,45 \%)$ fueron remitidos para valoración y manejo al servicio Cirugía Oral y Maxilofacial de sus respectivas entidades aseguradoras, debido a hallazgos clínicos y paraclínicos que sugerían compromiso óseo en cefalometría que pudiera estar en relación con el diagnóstico común de trastorno respiratorio del sueño. De los 422 pacientes referidos, 33 fueron sometidos a cirugía de avance máxilomandibular (7,8\%), 62 pacientes, a Osteotomía Sagital $(14,6 \%), 32$, a mentogenioplastia $(7,58 \%)$, mientras que 33 pacientes requirieron otro tipo de procedimiento quirúrgico por parte del servicio como parte del manejo integral del trastorno respiratorio del sueño $(7,8 \%)$.

Un total 160 pacientes $(37,91 \%)$, de 422 que fueron remitidos al servicio de cirugía oral y maxilofacial, fueron sometidos a algún tipo de cirugía maxilofacial como tratamiento definitivo o adyuvante para el trastorno respiratorio del sueño. (Gráfico 4)

Se encontró en promedio un IAH prequirúrgico de 35,5 por hora frente a un IAH postquirúrgico de 12,8 por hora. (Gráfico 5).

$\mathrm{El}$ registro promedio de saturación basal de $\mathrm{O} 2$ prequirúrgica fue de $88 \%$, frente a una saturación postoperatoria del $91,5 \%$.

Se encontró en promedio una saturación mínima de $\mathrm{O} 2$ prequirúrgica de $78,19 \%$ frente a una saturación mínima posterior a las intervenciones quirúrgicas del $86,13 \%$, siendo un resultado estadístico postoperatorio relevante como indicador de mejoría. (Gráfico 6 y 7).

En el estudio prequirúrgico de los pacientes sometidos a intervención operatoria única o múltiple, se encontró un promedio 29 microdespertares/hora durante el estudio polisomnográfico, con respecto a 7,9 microdespertares/hora como promedio de los estudios postoperatorios, vislumbrando una mejorando notable de la calidad del sueño.

En total, se presentaron complicaciones postoperatorias en 12 pacientes $(3,2 \%)$. La complicación más común fue hemorragia del lecho amigdalino en 8 pacientes $(66,6 \%)$, requiriendo revisión y corrección intraoperatoria con adecuada evolución clínica y de recuperación.

Otras de las complicaciones quirúrgicas evidenciadas fueron: Epistaxis en 1 paciente, Hematoma en lengua en 2 pacientes. Un paciente presentó edema traqueal asociado a hematoma disecante de cuello posterior a la realización de un procedimiento quirúrgico en la lengua submucosal minimally invasive lingual ecxision (SMILE); debido a las complicaciones derivadas de la vía área superior y extensivas a vía área inferior, fallece por falla ventilatoria.

Se realizó prueba de distribución normal de Shapiro wilk para una muestra de 256 pacientes, obteniendo una distribución no normal. Por lo que se utilizó prueba de Willcoxon para diferencia de medianas, de las muestras pareadas obteniendo una $p<0,00001$, lo cual muestra que hay una di- 


\section{Procedimientos realizados por Cirugía Oral y Maxilofacial para el tratamiento de SAHOS}
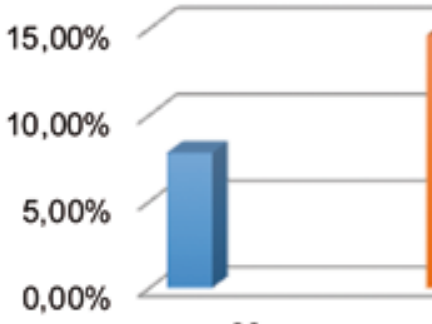

33

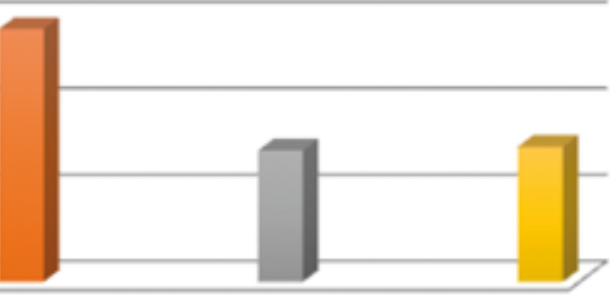

62

32

33

= AVANCE MAXILOMANDIBULAR

- OSTEOTOMIA SAGITAL

" MENTOGENIOPLASTÍA

$=$ OTROS

Gráfica 4. Procedimientos realizados por servicio de Cirugía Oral y Maxilofacial de forma extrainstitucional para el tratamiento del SAHOS.

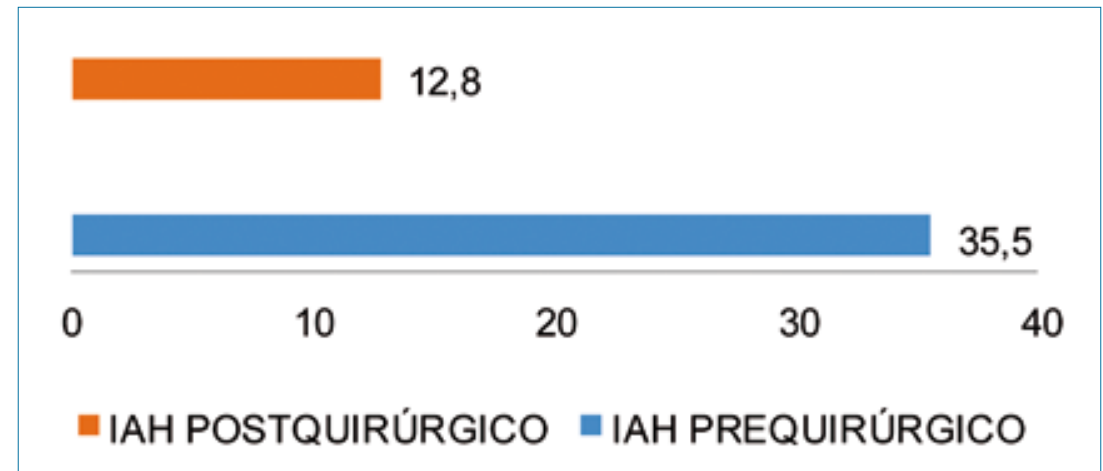

Gráfica 5. Comparación Índice de Apnea Hipopnea del sueño (IAH) Prequirúrgico y Postquirúrgico en pacientes sometidos a procedimientos quirúrgicos como tratamiento del trastorno respiratorio del sueño.

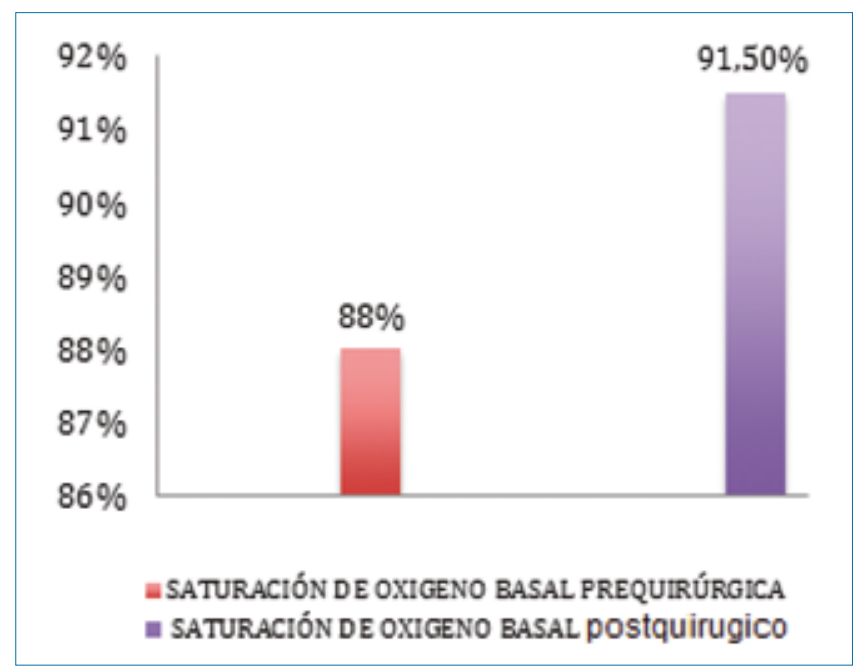

Gráfica 6. Comparación Índice de Saturación mínima de Oxigeno Prequirúrgico y Postquirúrgico en pacientes sometidos a procedimientos quirúrgicos como tratamiento del trastorno respiratorio del sueño.

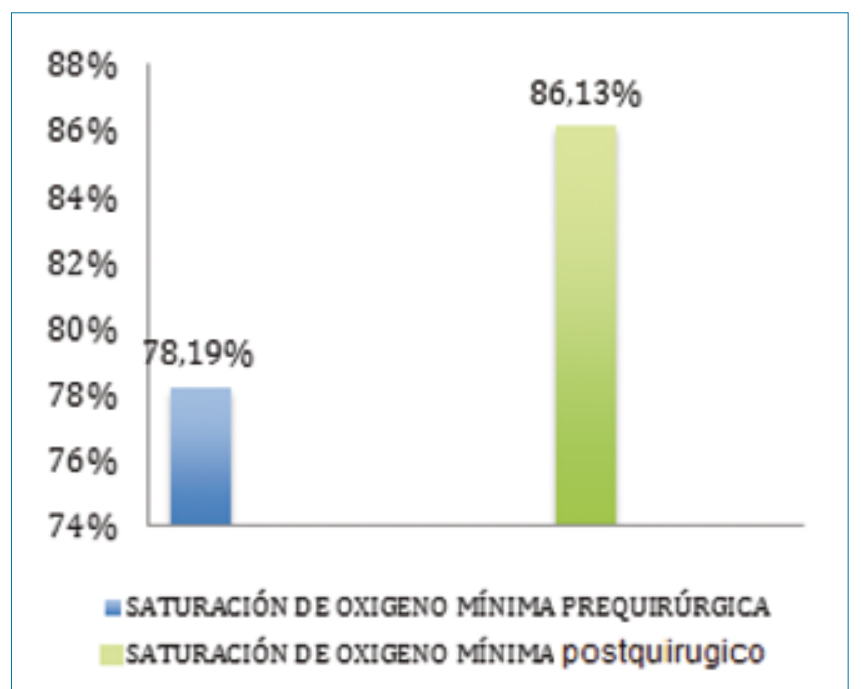

Gráfica 7. Comparación Índice de Saturación basal de Oxigeno Prequirúrgico y Postquirúrgico en pacientes sometidos a procedimientos quirúrgicos como tratamiento del trastorno respiratorio del sueño. 
ferencia estadísticamente significativa entre los resultados de IAH, índice de Saturación e índice de saturación de oxigeno basal y mínima prequirúrgicos y postquirúrgicos.

\section{Discusión}

La Clínica José A. Rivas, es un centro de remisión nacional de pacientes con diagnóstico de trastorno respiratorio del sueño quienes son remitidos por presentar dificultades en su tratamiento derivadas de rechazo del CPAP, o individuos que requieren cirugía con indicaciones claras clínicas y paraclínicas al igual que algunos pacientes que logran adaptarse al CPAP, que por sus comorbilidades la eficiencia de este no es suficiente, requieren de valoración y manejo quirúrgico, siendo entonces remitidos al centro especializado en mención. Debido a esto en un inicio se asumiría que el $100 \%$ de los pacientes remitidos terminarían en procedimientos quirúrgicos, pero gracias a un programa estricto de identificación y promoción de terapia de presión positiva se logra adaptar la mayor cantidad de pacientes y son pocos los que terminan en algún procedimiento quirúrgico siendo una de las principales indicaciones el mejorar la terapia de presión positiva y bajar el riesgo relativo.

Dentro de los hallazgos relevantes se puede valorar una consulta al centro especializado en apnea del sueño con una distribución por género muy similar, sin embargo los pacientes de sexo masculino son sometidos con mayor frecuencia a procedimientos quirúrgicos que buscan solucionar la obstrucción de la vía área superior, debido a que tienen menor adherencia al uso del dispositivo de presión positiva (CPAP). La posible explicación de las diferentes tasas de adherencia al CPAP como primera línea de tratamiento, es dada por la aceptación generalizada en mayor medida en el sexo femenino, que en términos generales cumplen acuciosamente y con mayor atención las diferentes recomendaciones que si son seguidas en su totalidad, incrementan radicalmente el éxito de adherencia al dispositivo.

Con respecto a la población pediátrica, es importante señalar que muchos de los procedimientos prequirúrgicos, que buscan identificar el patrón obstructivo de la vía aérea respiratoria superior, entre ellos y de forma primordial la nasofibrolaringoscopia, se obvió o no se realizó, debido a que el patrón de colapso se podía identificar en el examen físico, siendo la hipertrofia amigdalina la causa del SAHOS en muchos de ellos incluso en muchos a quienes se les realiza la fibronasolaringoscopia por no tener hipertrofia pero que terminan siendo la causa del colapso sin tener un aparente crecimiento de las mismas.

De forma comparativa, la tasa de éxito valorada subjetiva y objetivamente, para la muestra reclutada en el presente estudio, podría ser superior a la de otras instituciones que realizan cirugía para trastornos respiratorios del sueño, consideramos que esto es posible debido a que los pacientes son individualizados en cuanto a su patrón obstructivo que desencadena la patología de forma clínica (con una adecuado examen físico) y paraclínica (polisomografía, nasofibrola- ringoscopia, DISE, etc). De esta forma se individualiza el tratamiento con miras a resolver dicha obstrucción, y las cirugías indicadas son específicas para cada paciente, y con un fin resolutivo claro y no aleatorio. Esta individualización no sucede en otros centros, en donde los procedimientos quirúrgicos realizados son rutinariamente los mismos de manera reiterada, sin atender las necesidades específicas únicas del patrón obstructivo en cada paciente.

Es de resaltar, que del total de pacientes remitidos (6542), solo el 5,59\% son intervenidos quirúrgicamente con el fin de solucionar la obstrucción de la vía área superior, con criterios claros clínicos y paraclínicos, y en ocasiones con el fin de lograr una adecuada adaptación al dispositivo de presión positiva. El restante $94,41 \%$ logra una adecuada adaptación al CPAP, o este es sustituido por la tecnología adecuada luego de una exhaustiva y minuciosa revaloración que evita la necesidad de intervención quirúrgica. Estas tasas de adaptación a los dispositivos de presión positiva son muy elevadas y satisfactorias para el personal a cargo de la clínica de apnea.

\section{Conclusiones}

De manera global, el manejo quirúrgico en SAHOS disminuye de forma estadísticamente significativa la severidad del síndrome, en algunos casos logrando tasas de curación temporal, y en otros facilitando la adaptación a dispositivos de presión positiva como primera línea de manejo y mayor tasa de efectividad a largo plazo. Es posible evidenciar dicha mejoría, valorando el incremento del nivel de oxigenación tisular, calidad del sueño, y sensación de bienestar durante la vigilia, además de evitar el empeoramiento o avance de las enfermedades comorbidas o asociadas al SAHOS.

Las tasas de complicaciones derivadas de los procedimientos quirúrgicos de la vía aérea superior que buscan disminuir el grado de obstrucción pueden presentar una incidencia de complicaciones mínima, y cuando se presentan pueden ser resueltas en la inmensa mayoría de los casos.

Este estudio plantea la responsabilidad de diseñar otro de forma prospectiva para identificar los determinantes predictivos de éxito en nuestros protocolos al igual que estandarizar las técnicas de diagnóstico fibroscópico para que sean reproducibles.

\section{REFERENCIAS}

1. American Academy of Sleep Medicine. The International Classification of Sleep Disorders: Diagnostic and coding manual. 2a Ed. Westchester: American Academy of Sleep Medicine; 2005. p. 298.

2. Cummings CW. Otolaryngology-Head and Neck surgery. $5^{\text {a }}$ Ed. Filadelfia, PA: Mosby Elsevier; 2010. p. 2956.

3. Aurora R, Casey KR, Kristo D, Auerbach S, Bista SR, Chowdhuri S. Practice Parameters for the Surgical Modifications of the Upper Airway for Obstructive Sleep Apnea in Adults. Sleep. 2010; 33(10): 1408-13. 
4. Camacho M, Jacobson RL, Schendel S. Surgical Treatment of Obstructive Sleep Apnea. 2013; 8(4): 495-503.

5. American Academy of Sleep Medicine Task Force. Sleeprelated breathing disorders in adults: recommendations for syndrome definition and measurement techniques in clinical research; the report of an American Academy of Sleep Medicine task force. Sleep. 1999; 22(5): 667-689.

6. Lanfranco F. Sleep apnea syndrome and hypothyroidism. Endocrine [Internet]. 2013 [Citado 2014 Feb 25]; 44(3): 551-2. Disponible en: http://www.ncbi.nlm.nih.gov/ pubmed/24114404

7. Woodson BT, Franco R. Physiology of sleep disordered breathing. Otolaryngol Clin North Am [Internet]. 2007 [Citado: 2014 Feb 25];40(4): 691-711. Disponible en: http:// www.ncbi.nlm.nih.gov/pubmed/17606019

8. Carvalho B, Hsia J, Capasso R. Surgical therapy of obstructive sleep apnea: a review. Neurotherapeutics [Internet]. 2012 [Citado 2014 Mar 6]; 9(4): 710-6. Disponible en: http://www. ncbi.nlm.nih.gov/pmc/articles/PMC3480570/pdf/13311_2012_ Article_141.pdf

9. Pérez PV, Tobar LN. Evaluacion de la Via Aerea Superior en Pacientes con SAHOS Mediante Cefalometria 3D y Multiplanar. Acta otorrinolaringol. cir. cabeza cuello. 2012; 40(1): 42-47.

10. Goodday R. Diagnosis, treatment planning, and surgical correction of obstructive sleep apnea. J Oral Maxillofac Surg [Internet]. 2009 [Citado: 2014 Feb 25]; 67(10): 2183-96. Available from: http://www.ncbi.nlm.nih.gov/ pubmed/19761912

11. Mete T, Yalcin Y, Berker D, Ciftci B, Guven Firat S, Topaloglu $\mathrm{O}$, et al. Relationship between obstructive sleep apnea syndrome and thyroid diseases. Endocrine [Internet]. 2013 [Citado 2014 Feb 25]; 44(3): 723-8. Disponible en: http:// www.ncbi.nlm.nih.gov/pubmed/23564558

12. Friedman M, Maley A, Kelley K, Leesman C, Patel A, Pulver $\mathrm{T}$, et al. Impact of nasal obstruction on obstructive sleep apnea. Otolaryngol Head Neck Surg [Internet]. 2011 [Citado 2014 Mar 7];144(6):1000-4. Disponible en: http://www.ncbi. nlm.nih.gov/pubmed/21493302
13. MacKay SG, Carney AS, Woods C, Antic N, McEvoy RD, Chia M. Modified Uvulopalatopharyngoplasty and Coblation Channeling of the Tongue for Obstructive Sleep Apnea: A Multi-Centre Australian Trial. J Clin Sleep Med. 2013; 9(2):117-24.

14. Braga A, Grechi TH, Eckeli A, Vieira BB, Itikawa CE, Küpper DS, et al. Predictors of uvulopalatopharyngoplasty success in the treatment of obstructive sleep apnea syndrome. Sleep Med. 2013; 14(12): 1266-71.

15. Caples SM, Rowley JA, Prinsell JR, Pallanch JF, Elamin MB, Katz SG, et al. Surgical Modifications of the Upper Airway for Obstructive Sleep Apnea in Adults: A Systematic Review and Meta-Analysis. Sleep. 2010; 33(10): 1396-407.

16. Choi JH, Kim S-N, Cho JH. Efficacy of the Pillar implant in the treatment of snoring and mild-to-moderate obstructive sleep apnea: a meta-analysis. Laryngoscope [Internet]. 2013 [Citado 2014 Mar 7]; 123(1):269-76. Disponible en: http:// www.ncbi.nlm.nih.gov/pubmed/22865236

17. Friedman M, Ibrahim H, Bass L. Clinical staging for sleepdisordered breathing. Otolaryngol Head Neck Surg. 2002; 127(1): 13-21.

18. Engleman HM, Wild MR. Improving CPAP use by patients with the sleep apnoea/hypopnoea syndrome (SAHS). Sleep Med Rev. 2003; 7(1): 81-99.

19. Han F, Song W, Li J, Zhang L, Dong X, He Q. Influence of UPPP surgery on tolerance to subsequent continuous positive airway pressure in patients with OSAHS. Sleep Breath. 2006; 10(1): 37-42.

20. Friedman M, Lin HC, Gurpinar B, Joseph NJ. Minimally invasive single-stage multilevel treatment for obstructive sleep apnea/ hypopnea syndrome. Laryngoscope. 2007; 117(10): 1859-63.

21 . Verse T. Update on surgery for obstructive sleep apnea syndrome. HNO. 2008; 56(11): 1098-1104

22. Lin HC, Friedman M, Chang HW, Gurpinar B. The efficacy of multilevel surgery of the upper airway in adults with obstructive sleep apnea/hypopnea syndrome. Laryngoscope. 2008; 118(5): 902-8.

23. Sher AE, Schechtmann KB, Piccirillo JF. The efficiency of surgical modifications of the upper airway in adults with obstructive sleep apnea syndrome. Sleep. 1996; 19(2): 156-77. 